Service social

\title{
Aide ou contrôle. L'intervention thérapeutique sous contrainte, Claude Seron et Jean-Jacques Wittezaele, Belgique, Éd. de Boeck-Wesmael, 1991, 367 pages. Distribué au Canada par les Éditions du renouveau pédagogique, Montréal.
}

\section{Suzanne Lemire}

Volume 41, numéro 3, 1992

Intervenir en contexte d'autorité

URI : https://id.erudit.org/iderudit/706588ar

DOI : https://doi.org/10.7202/706588ar

Aller au sommaire du numéro

Éditeur(s)

École de service social de l'Université Laval

ISSN

1708-1734 (numérique)

Découvrir la revue

Citer ce compte rendu

Lemire, S. (1992). Compte rendu de [Aide ou contrôle. L'intervention thérapeutique sous contrainte, Claude Seron et Jean-Jacques Wittezaele, Belgique, Éd. de Boeck-Wesmael, 1991, 367 pages. Distribué au Canada par les Éditions du renouveau pédagogique, Montréal.] Service social, 41(3), 125-126. https://doi.org/10.7202/706588ar d'utilisation que vous pouvez consulter en ligne. 


\section{RECENSIONS}

\section{AIDE OU CONTRÔLE.}

\section{L'INTERVENTION THÉRAPEUTIQUE SOUS CONTRAINTE}

Claude Seron et Jean-Jacques Wittezaele,

Belgique, Éd. de Boeck-Wesmael, 1991, 367 pages.

Distribué au Canada par les Éditions du renouveau pédagogique, Montréal.

L'ouvrage de Seron et Wittezaele démontre l'application de l'approche «thérapie brève » au secteur de la protection de la jeunesse. On fait ici référence à la thérapie brève développée au Mental Research Institute de Palo Alto (en Californie) par Batesen, Jackson, Watzlawick, Fish et Weakland. L'approche a été expérimentée à Liège au Centre liégeois d'intervention familiale (CLIF), centre responsable entre autres d'appliquer des "mesures de guidance » ordonnées par le tribunal des mineurs et leur famille.

Après avoir décrit en introduction différentes contraintes du contexte judiciaire, les auteurs discutent au premier chapitre - «La systémique : un nouveau paradigme » - des prémisses épistémologiques, de l'approche systémique et la thérapie familiale, de même que de l'induction du changement dans le système familial. Le deuxième chapitre définit le " contexte contraignant » en précisant les notions de contexte et d'intervention sous contrainte judiciaire. Les trois chapitres suivants portent sur les assises théoriques de la " thérapie brève ", le développement du Centre de Palo Alto, les principes généraux, les prémisses, les méta-questions de l'intervention, les stratégies et tactiques de changement ainsi que sur l'évaluation de l'intervention. Plus précisément, au cinquième chapitre, les auteurs décrivent la réflexion effectuée pour décider de recourir à la thérapie brève.

Les sixième et septième chapitres, "Le modèle revisité " et " La thérapie brève sous contrainte judiciaire », envisagent les caractéristiques du modèle appliqué à son nouveau contexte et les nouvelles étapes du modèle 
de Palo Alto ainsi « revisité ». Des thèmes tels que le paradoxe de l'aide contrainte, l'établissement d'une relation complémentaire avec un usager réticent, les stratégies paradoxales y sont discutés. Les auteurs se demandent aussi : " Mais qui donc a un problème ? Vaut-il mieux prévenir que guérir ? ». Ces deux chapitres, cœur de l'ouvrage (p. 189 à 295), sont très pragmatiques.

En conclusion, les auteurs se penchent sur les grandes faiblesses des théories psychologiques et sur l'intérêt de l'application du modèle de thérapie brève. Ils insistent sur la position « méta » indispensable à l'intervention systémique et sur la nécessité pour l'intervenant « de conserver sa liberté de manœuvre par rapport aux moyens à mettre en œuvre pour résoudre les problèmes », ajoutant que " ...I'approche systémique et la thérapie brève nous ont libérés d'un sentiment de confusion assez typique des situations de double contrainte, tout en offrant des moyens d'intervention plus efficace » (p. 306).

Quatre annexes et une bibliographie terminent le livre. Les annexes sont des schémas, outils à l'intervention : La modélisation de la programmation du changement en analyse systémique ; Le questionnement pour faire préciser le problème ; Les stratégies de changement en thérapie brève ; La position du client.

Intéressant, ce livre qui propose un modèle d'intervention dans un contexte de contrainte, dépassant ainsi l'idée que l'aide n'est pas possible dans un contexte de contrôle. Bien que les auteurs soient européens, leurs propositions sont applicables chez nous. Plusieurs illustrations de situation tout au long de l'ouvrage et en conclusion facilitent la compréhension et rendent la lecture très vivante. L'humour est aussi présent dans ce livre, probablement à cause de l'influence de l'application du modèle chez les auteurs. Nous y voyons une réponse aux sentiments d'impuissance souvent vécus par les intervenants en protection de la jeunesse. À l'instar des auteurs, toutefois, nous croyons qu'une mise en garde s'impose quant au recours à certaines techniques sans la compréhension théorique du modèle, des valeurs véhiculées et des différentes techniques utilisées.

Dans la foulée des recommandations du rapport «La protection sur mesure » (Harvey II) qui favorise notamment une intervention précoce, intensive et brève, il serait heureux que les intervenants expérimentent I'application de ce modèle. Nous le croyons utile pour une bonne partie de notre clientèle. Pourquoi pas des îlots d'expérimentation dans les différentes directions de la protection de la jeunesse et un lieu commun de formation, d'appui à l'expérimentation et à l'évaluation?

Suzanne LEMIRE

Travailleuse sociale

Centre de services sociaux du Centre-du-Québec 